\title{
Dietary intake of carbohydrates and risk of type 2 diabetes: the European Prospective Investigation into Cancer-Norfolk study
}

\author{
Sara Ahmadi-Abhari ${ }^{1 *}$, Robert N. Luben ${ }^{1}$, Natasha Powell ${ }^{1}$, Amit Bhaniani ${ }^{1}$, Rajiv Chowdhury ${ }^{1}$, \\ Nicholas J. Wareham ${ }^{2}$, Nita G. Forouhi ${ }^{2}$ and Kay-Tee Khaw ${ }^{1}$ \\ ${ }^{1}$ Department of Public Health and Primary Care, University of Cambridge, Strangeways Research Laboratory, \\ Worts Causeway, Cambridge CB1 $8 R N$, UK \\ ${ }^{2}$ MRC Epidemiology Unit, Institute of Metabolic Sciences, Cambridge, UK
}

(Submitted 14 November 2012 - Final revision received 15 May 2013 - Accepted 24 May 2013 - First published online 23 July 2013)

\section{Abstract}

In the present study, we investigated the association between dietary intake of carbohydrates and the risk of type 2 diabetes. Incident cases of diabetes ( $n$ 749) were identified and compared with a randomly selected subcohort of 3496 participants aged $40-79$ years. For dietary assessment, we used $7 \mathrm{~d}$ food diaries administered at baseline. We carried out modified Cox proportional hazards regression analyses and compared results obtained from the different methods of adjustment for total energy intake. Dietary intakes of total carbohydrates, starch, sucrose, lactose or maltose were not significantly related to diabetes risk after adjustment for confounders. However, in the residual method for energy adjustment, intakes of fructose and glucose were inversely related to diabetes risk. The multivariable-adjusted hazard ratios (HR) of diabetes comparing the extreme quintiles of intake were $0.79(95 \% \mathrm{CI} 0.59,1.07 ; P$ for trend $=0.03)$ for glucose and $0.62(95 \% \mathrm{CI} 0.46$, $0.83 ; P$ for trend $=0.01$ ) for fructose. In the nutrient density method, only fructose was inversely related to diabetes risk (HR $0.65,95 \% \mathrm{CI}$ $0 \cdot 48,0 \cdot 88)$. The replacement of $5 \%$ energy intake from SFA with an isoenergetic amount of fructose was associated with a $30 \%$ lower diabetes risk (HR 0.69, 95\% CI 0.50, 0.96). Results of the standard and energy partition methods were similar to those of the residual method. These prospective findings suggest that the intakes of starch and sucrose are not associated, but that those of fructose and glucose are inversely associated with diabetes risk. Whether the inverse associations with fructose and glucose reflect the effect of substitution of these carbohydrate subtypes with other nutrients (i.e. SFA), their net higher intake or other nutrients associated with their intake remains to be established through further investigation.

Key words: Type 2 diabetes: Carbohydrates: Nutrition: Case-cohort studies

The identification of modifiable risk factors for type 2 diabetes, such as dietary factors, plays an important role in the prevention of this condition ${ }^{(1)}$. Carbohydrates are the main dietary factors affecting insulin secretion and postprandial glycaemia $^{(2)}$. Evidence from observational studies indicates that higher glycaemic index (a measure used to quantify glycaemic response to carbohydrates in food) and glycaemic load (glycaemic index multiplied by the quantity of carbohydrates in food) are associated with an increased risk of incident type 2 diabetes $^{(3)}$. However, the role of dietary intake of carbohydrates and their subtypes (such as starch, sucrose, glucose and fructose), either in combination or in isolation, in the development of type 2 diabetes remains unclear. Several cohort studies have found a positive association between the risk of diabetes and total carbohydrates ${ }^{(4-6)}$ and $\operatorname{starch}^{(4,7)}$, while others have found a non-significant inverse association $^{(8-10)}$. Few studies have found a significant or non-significant inverse association of diabetes risk with total sugars $^{(7,9)}$ and sucrose ${ }^{(8-10)}$, while others have reported a non-significant positive association ${ }^{(4,11,12)}$. Furthermore, two studies have found a strong positive association of diabetes risk with glucose and fructose ${ }^{(8,11)}$, while others have reported no association ${ }^{(6,7)}$.

The discrepancy between results of different studies for the same carbohydrate subtype might have resulted from the method used for dietary assessment. All the previous studies have used the FFQ for dietary assessment. Inconsistent findings might result from using different FFQ, which are restricted to a limited number of dietary items and may capture different (and not all) sources of carbohydrates in different studies. On the other hand, inconsistent results for different carbohydrate subtypes (e.g. the same study reporting a positive association of diabetes risk with glucose and fructose and an inverse association with sucrose ${ }^{(8)}$ ) might result

Abbreviations: EPIC, European Prospective Investigation into Cancer; HR, hazard ratio.

*Corresponding author: S. Ahmadi-Abhari, fax +44 1223 740177, email sa540@medschl.cam.ac.uk 
from different types of carbohydrates having different metabolic effects ${ }^{(9,13)}$.

The aim of the present study was to investigate the association between dietary intake of different carbohydrate subtypes and the risk of developing type 2 diabetes in a prospective cohort study using the food diary method for dietary assessment.

\section{Methods}

\section{Study participants}

We conducted a case-cohort study in the context of the European Prospective Investigation into Cancer (EPIC)-Norfolk study. The detailed design of the ongoing EPIC-Norfolk cohort study has been described previously ${ }^{(14)}$. Briefly, 25639 men and women, aged 40-79 years, who lived in Norfolk, England, were recruited into the study, and they underwent the first (baseline) health examination between 1993 and 1997. The participants were followed up using a postal health questionnaire during 2002-2004, a second health check-up at 3-5 years (1998-2000) and a further postal questionnaire at 10 years after recruitment into the study. The present study was conducted according to the guidelines laid down in the Declaration of Helsinki, and all procedures involving human subjects were approved by the Norfolk Local Research Ethics Committee. All the volunteers gave written informed consent. Cases were study participants with a confirmed diagnosis of incident type 2 diabetes during follow-up. Since nutritional information from food diaries was not collected for the entire cohort, a subcohort of 4000 participants were randomly chosen as controls from all the participants who were recruited into the EPIC-Norfolk study.

Participants were excluded from the present study if they had a self-reported doctor-diagnosed history of prevalent diabetes, cancer, myocardial infarction and cerebrovascular accident (stroke) at baseline health examination or if their food diary data were not available.

Incident cases of type 2 diabetes were ascertained by self-report of diabetes diagnosed by a physician or new administration of diabetes medication at follow-up, which was verified by medical records or by record linkage with external sources independent of the individuals' participation in a follow-up health examination or response to the postal questionnaires. External sources used to ascertain diabetes cases included listing with general practice diabetes registers, local hospital outpatient diabetes registers, hospital admission information that screened for diabetes-related conditions and Office for National Statistics mortality data with coding for diabetes. Possible cases solely based on self-report, and not confirmed by another data source, did not qualify as confirmed cases of diabetes and were not included in the present study. Cases ascertained up to 31 July 2006 were included.

Among the 892 confirmed incident cases of diabetes recorded until July 2006, 123 cases were excluded due to reporting of a history of cancer ( $n$ 51), myocardial infarction ( $n$ 56) and cerebrovascular accident ( $n$ 24) at baseline, three were excluded due to unavailability of food diary data and seventeen were excluded due to missing data on covariates, leaving 749 cases for the present analysis. From the 4000 participants randomly selected, 455 were excluded due to reporting of a history of cancer ( $n$ 225), myocardial infarction ( $n$ 109), cerebrovascular accident ( $n$ 45) and diabetes ( $n$ 121) at baseline, fourteen were excluded due to unavailability of food diary data and thirty-five were excluded due to missing data on covariates. Among the 3496 subcohort participants who remained for the analysis, $129(3.7 \%)$ developed diabetes.

\section{Assessment of dietary exposures}

The dietary exposure variables were carbohydrate subtypes including starch, individual sugars (sucrose, glucose, fructose, lactose and maltose), total sugars (sum of individual sugars) and total carbohydrates (sum of starch and total sugars).

The $7 \mathrm{~d}$ food diary was used for dietary assessment of nutrients (i.e. carbohydrate subtypes, fat and protein) and total energy intake in the present study. The methods used for dietary assessment in the EPIC-Norfolk study have been described in detail previously ${ }^{(15)}$. Briefly, the $7 \mathrm{~d}$ food diary comprised a booklet in which the description and amount of foods consumed at main meals and between meal times over seven consecutive days could be recorded ${ }^{(15)}$. Convenient household measures such as tablespoons, bowls, glasses, numbers, slices or packet weights and seventeen sets of colour photographs were used to describe portion sizes.

The nutrient content of each item was derived by matching the descriptions in the $7 \mathrm{~d}$ food diary with food composition tables that provide information on the amount of nutrients in each item using a data entry system called Data Into Nutrients for Epidemiological Research (DINER) ${ }^{(16)}$. Nutrient intakes were computed by multiplying the portion size by the nutrient content of each item, summed for each person for each day, and averaged across the number of days the diary was kept ${ }^{(14,15)}$. The average number of days the food diaries were kept for both cases and non-cases was 6.5 (SD 1.6) $\mathrm{d}$, and over $90 \%$ had kept the diary for all the $7 \mathrm{~d}$.

\section{Assessment of non-dietary exposures}

During the clinic visit, trained nurses took anthropometric measurements of individuals in light clothing without shoes. Height was measured to the nearest millimetre using a freestanding stadiometer, and weight was measured to the nearest $100 \mathrm{~g}$ using a digital scale. BMI was calculated as weight in $\mathrm{kg}$ divided by the square of height in metres ${ }^{(14)}$.

Family history of diabetes, smoking status (current, former and never), alcohol consumption (units per week) and other lifestyle factors were assessed as part of a health and lifestyle questionnaire administered at baseline examination. A four-point physical activity index based on occupational and non-occupational physical activities was used to categorise participants into four groups: inactive; moderately inactive; moderately active; active. Educational status was based on the highest qualification attained and was categorised into four groups: degree or equivalent; A-level (educational attainment 
by the age of 17 years); O-level (usual minimal school-leaving age of 15 years); less than O-level or no qualifications ${ }^{(14)}$.

\section{Statistical analyses}

The hazard ratio (HR) of diabetes was calculated using a modified Cox proportional hazards regression analysis that takes account of the design of the case-cohort study. This method is a weighted Cox regression introduced by Prentice $^{(17,18)}$. Person-years at risk were calculated from the date of recruitment until diagnosis of diabetes, death, loss to follow-up or end of follow-up (end of July 2006), whichever was first. To explore the association between the intake of carbohydrate subtypes and the risk of diabetes, we used and compared the results of different methods for adjustment for total energy intake: (1) standard method; (2) residual method; (3) nutrient density method; (4) energy partition method $^{(19-21)}$. Nutrient values were treated separately as continuous variables (HR of diabetes was calculated per standard deviation higher intake of each nutrient) and categorical variables (HR of diabetes was calculated for quintiles of the nutrient values). Standard deviations and cut-offs for the quintiles of nutrient intakes were calculated based on the distribution in the representative subcohort sample. The nutrient values entered as exposure variables were $\mathrm{g} / \mathrm{d}$ of intake in the standard method, percentage of energy intake from each nutrient (nutrient density) in the nutrient density method and the energy-adjusted intake of each nutrient in the residual method. The energy-adjusted values for each nutrient (for the residual method) were calculated by adding the residuals of regressing the nutrient on total energy intake to the mean intake of that nutrient. In these three methods, total energy intake was entered into the model as a covariate. The HR calculated from this model can be interpreted as the effect of substituting the exposure variable with other energyproducing nutrients that are not included in the model while maintaining a constant total energy intake. In the energy partition method, the energy intake from a nutrient was entered into the model and energy intake from all other nutrients was entered as a covariate. The HR calculated from this model can be interpreted as the net effect of a higher intake of the nutrient ${ }^{(19-21)}$.

We conducted categorical analyses using all the methods because the methods are not equivalent in categorical analyses. For example, a person in the fifth quintile of glucose intake is not necessarily in the fifth quintile of percentage of energy intake from glucose ${ }^{(19-21)}$.

Other covariates in all the multivariable analyses were age, sex, BMI, family history of diabetes (yes/no), physical activity (four categories), smoking (three categories), units of alcohol intake per week and level of education (as a proxy for socioeconomic status, four categories). Robust standard errors were used to calculate $95 \%$ CI.

A series of multivariable nutrient density models were further constructed to statistically model specific substitution patterns with protein, fat and fatty acids (SFA, MUFA and PUFA) associated with diabetes risk. The coefficient obtained from these models can be interpreted as the HR of diabetes associated with the substitution of $5 \%$ energy intake from each nutrient for other carbohydrate subtypes, protein, fat or fatty acids in the relevant model. Information on trans-fatty acids was not available.

In sensitivity analyses, we excluded individuals who developed diabetes within 5 years of recruitment into the study, repeated analyses in men and women separately, and after excluding potential energy misreporters (participants belonging to the highest and lowest $5 \%$ of the energy intake: BMR ratio category). Effect modification by BMI was tested on the multiplicative scale using the Wald test. Similarly, effect modification by family history of diabetes was also tested using the same method.

All statistical analyses were conducted using Stata 10.0 (StataCorp, 2007, Stata Statistical Software: Release 10; StataCorp LP). The level of statistical significance for all the analyses was chosen at $\alpha=0.05$.

\section{Results}

The age and sex distribution of the 749 cases included in the present analysis was similar to that of all the 892 cases (data not shown). The mean age of diabetes cases was $61 \cdot 2$ (SD 8.3) years, and 56.5\% were men (Table 1). The mean and median durations of follow-up from baseline assessment until diagnosis of diabetes were 6.3 (SD 2.5) and 6.2 years, respectively. Participants who subsequently developed diabetes were more likely to be obese, hypertensive, less physically active and less educated compared with the representative subcohort members who did not develop diabetes (Table 1). A higher proportion of subsequent cases of diabetes reported being on a weight reduction diet for obesity and tended to report slightly less alcohol ingestion, almost equal total energy intake, lower intake of carbohydrates, and higher intake of protein and fat compared with the subcohort members who did not subsequently develop diabetes. Participants in the subcohort who had a higher percentage of energy intake from total carbohydrates (Table 2) were more likely to be female, tended to be older, were slightly leaner, were less likely to smoke, had lower levels of alcohol intake, were more physically active, were more likely to have a family history of diabetes and had a lower total energy intake.

\section{Standard and residual methods for energy adjustment}

In the residual method, the energy-adjusted intake of each nutrient $(\mathrm{g} / \mathrm{d})$ was entered into separate models as a continuous variable and was adjusted for total energy intake. The HR of diabetes was inversely associated with a standard deviation higher intake of total carbohydrates, total sugars, sucrose, glucose and fructose in models adjusted for age and sex (Fig. 1). However, in multivariable analyses, total sugars and sucrose were no longer associated with diabetes risk, but there was a significant inverse association of diabetes risk with fructose and a borderline significant inverse association with glucose and total carbohydrates (Fig. 1).

In categorical analyses involving quintiles of intake of each nutrient, fructose and glucose showed an inverse association 
Table 1. Baseline characteristics of cases of type 2 diabetes and non-cases in the representative subcohort

(Mean values and standard deviations; numbers and percentages)

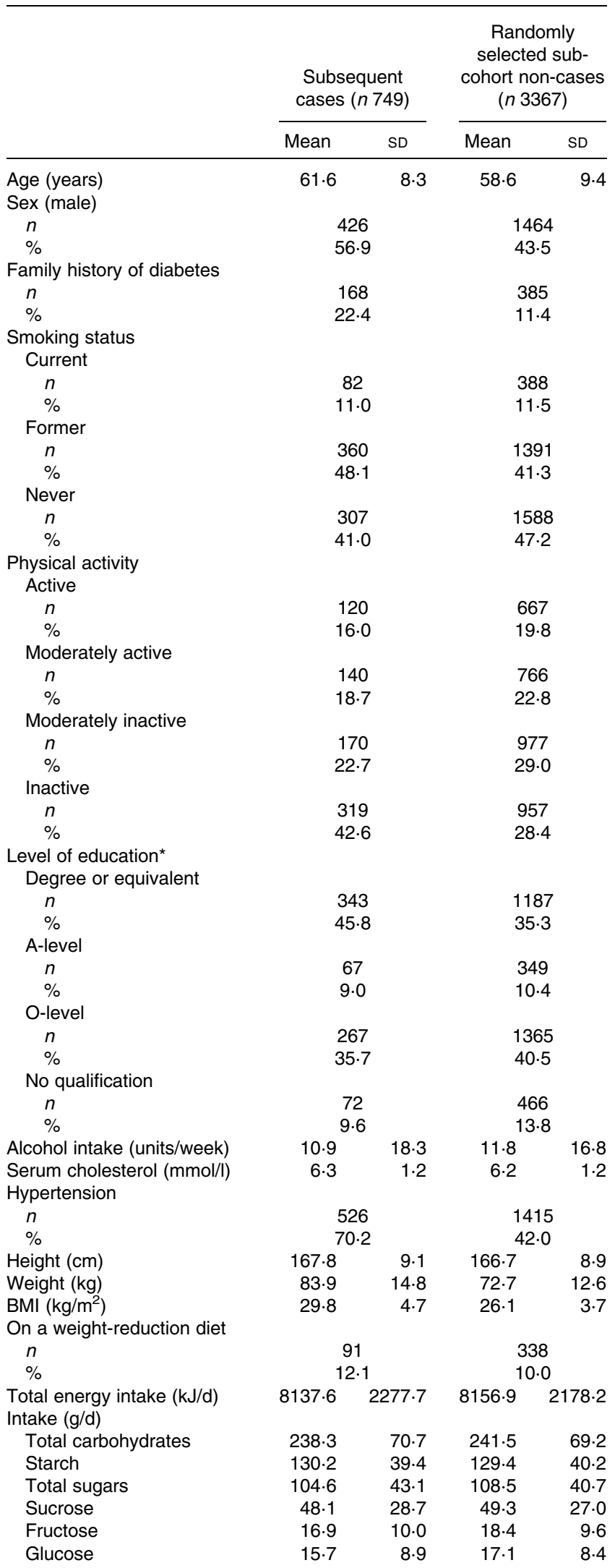

Table 1. Continued

\begin{tabular}{|c|c|c|c|c|}
\hline & \multicolumn{2}{|c|}{$\begin{array}{l}\text { Subsequent } \\
\text { cases }(n 749)\end{array}$} & \multicolumn{2}{|c|}{$\begin{array}{c}\text { Randomly } \\
\text { selected sub- } \\
\text { cohort non-cases } \\
(n 3367)\end{array}$} \\
\hline & Mean & SD & Mean & SD \\
\hline Lactose & $20 \cdot 1$ & $10 \cdot 1$ & $20 \cdot 2$ & $9 \cdot 3$ \\
\hline Maltose & 3.5 & $5 \cdot 3$ & $3 \cdot 1$ & 4.7 \\
\hline Protein & 74.9 & $19 \cdot 1$ & $72 \cdot 9$ & $18 \cdot 1$ \\
\hline Fat & $75 \cdot 7$ & $27 \cdot 1$ & $75 \cdot 0$ & $25 \cdot 7$ \\
\hline \multicolumn{5}{|c|}{$\begin{array}{l}\text { Percentage of total energy } \\
\text { intake (nutrient density \%) }\end{array}$} \\
\hline Total carbohydrates & $46 \cdot 2$ & $6 \cdot 6$ & $46 \cdot 6$ & $6 \cdot 5$ \\
\hline Starch & $25 \cdot 4$ & $4 \cdot 8$ & $25 \cdot 0$ & $4 \cdot 7$ \\
\hline Total sugars & $20 \cdot 1$ & $5 \cdot 9$ & $20 \cdot 9$ & $5 \cdot 8$ \\
\hline Sucrose & $9 \cdot 1$ & $4 \cdot 3$ & $9 \cdot 3$ & 4.0 \\
\hline Fructose & $3 \cdot 3$ & 1.9 & 3.6 & 1.9 \\
\hline Glucose & $3 \cdot 1$ & $1 \cdot 6$ & $3 \cdot 3$ & 1.5 \\
\hline Lactose & 3.9 & 1.7 & 3.9 & $1 \cdot 7$ \\
\hline Maltose & 0.6 & 0.8 & 0.6 & 0.8 \\
\hline Protein & $15 \cdot 8$ & 3.1 & $15 \cdot 3$ & $2 \cdot 7$ \\
\hline Fat & $34 \cdot 6$ & 5.9 & $34 \cdot 3$ & $5 \cdot 7$ \\
\hline
\end{tabular}

* Educational status was based on the highest qualification attained and was categorised into four groups: degree or equivalent; A-level (educational attainment by the age of 17 years); O-level (usual minimal school-leaving age of 15 years); less than O-level or no qualifications.

with diabetes risk. The multivariable-adjusted HR of diabetes comparing the extreme quintiles of glucose intake was 0.79 (95\% CI 0.59, 1.07; $P$ for trend $=0.03$ ) and the corresponding HR for fructose was 0.62 (95\% CI $0.46,0.83 ; P$ for trend $=0 \cdot 01$ ). Sucrose, starch and other carbohydrate subtypes were not significantly related to diabetes risk after adjustment for all covariates.

The standard method for energy adjustment produced results similar to those of the residual method (data not shown).

\section{Nutrient density method for energy adjustment}

In this set of analyses, quintiles of percentage of energy intake from each nutrient (nutrient density) were entered into the model and adjusted for total energy intake. The HR of diabetes exhibited a positive association with nutrient density for starch and an inverse association with nutrient density for sucrose, glucose, fructose and maltose in models adjusted for age and sex (Fig. 2). However, after adjustment for all covariates, only fructose remained significantly associated with an inverse risk of diabetes in the nutrient density method for energy adjustment (Fig. 2).

In statistical modelling of specific substitution patterns using nutrient density models (Table 3), we found that replacement of fructose with other carbohydrates was not significantly associated with an altered risk of type 2 diabetes. However, replacement of $5 \%$ energy intake from SFA with an isoenergetic amount of fructose was significantly associated with a $30 \%$ lower diabetes risk (HR 0.69, $95 \%$ CI 0.50, 0.96). Substitution of carbohydrates with PUFA and MUFA did not alter diabetes risk. The $\mathrm{HR}$ of diabetes for replacing 5\% energy intake from protein with an isoenergetic amount of fructose was 0.72 (95\% CI 0.51, 1.03). The replacement of other carbohydrates, protein or fatty acids with an isoenergetic amount of glucose did not significantly alter diabetes risk (Table 3). 
Table 2. Baseline characteristics of subsequent cases of diabetes and non-cases by quintiles of energy intake from total carbohydrates

(Mean values and standard deviations; numbers and percentages)

\begin{tabular}{|c|c|c|c|c|c|c|c|c|c|c|c|}
\hline \multirow{3}{*}{$\begin{array}{l}\text { Percentage of total energy intake from } \\
\text { carbohydrates (nutrient density \%) }\end{array}$} & \multicolumn{2}{|c|}{ Q1 } & \multicolumn{2}{|c|}{ Q2 } & \multicolumn{2}{|c|}{ Q3 } & \multicolumn{2}{|c|}{ Q4 } & \multicolumn{2}{|c|}{ Q5 } & \multirow[b]{3}{*}{$P$} \\
\hline & \multicolumn{2}{|c|}{$20-44 \cdot 1 \%$} & \multicolumn{2}{|c|}{$44 \cdot 2-48 \cdot 3 \%$} & \multicolumn{2}{|c|}{$48 \cdot 4-51 \cdot 6 \%$} & \multicolumn{2}{|c|}{$51.7-55.4 \%$} & \multicolumn{2}{|c|}{$55 \cdot 5-74 \%$} & \\
\hline & Mean & SD & Mean & SD & Mean & SD & Mean & SD & Mean & SD & \\
\hline \multicolumn{12}{|l|}{ Diabetes cases $(n 749)$} \\
\hline$n$ & \multicolumn{2}{|c|}{154} & \multicolumn{2}{|c|}{163} & \multicolumn{2}{|c|}{144} & \multicolumn{2}{|c|}{156} & \multicolumn{2}{|c|}{132} & \\
\hline Age (years) & $59 \cdot 8$ & $8 \cdot 7$ & $63 \cdot 2$ & $7 \cdot 3$ & $61 \cdot 7$ & $8 \cdot 7$ & $62 \cdot 8$ & $8 \cdot 3$ & $60 \cdot 6$ & $8 \cdot 1$ & 0.001 \\
\hline Sex (men) & & & & & & & & & & & $<0.001$ \\
\hline$n$ & \multicolumn{2}{|c|}{109} & \multirow{2}{*}{\multicolumn{2}{|c|}{$\begin{array}{c}100 \\
23.5\end{array}$}} & \multirow{2}{*}{\multicolumn{2}{|c|}{$\begin{array}{c}84 \\
19.7\end{array}$}} & \multirow{2}{*}{\multicolumn{2}{|c|}{$\begin{array}{c}70 \\
16.4\end{array}$}} & & & \\
\hline$\%$ & & & & & & & & & & & \\
\hline $\mathrm{BMI}\left(\mathrm{kg} / \mathrm{m}^{2}\right)$ & $30 \cdot 2$ & 4.7 & 29.4 & 4.5 & $30 \cdot 0$ & $5 \cdot 0$ & $30 \cdot 0$ & 4.7 & $29 \cdot 2$ & 4.5 & 0.32 \\
\hline Height & $170 \cdot 4$ & 9.5 & 167.9 & $8 \cdot 3$ & $168 \cdot 3$ & 9.7 & $166 \cdot 3$ & $9 \cdot 0$ & $166 \cdot 2$ & 8.7 & $<0.001$ \\
\hline Weight & 87.7 & $15 \cdot 2$ & 83.1 & 14.5 & 84.7 & $14 \cdot 8$ & 83.0 & 14.9 & $80 \cdot 7$ & $14 \cdot 0$ & $<0.001$ \\
\hline Smoking status (current) & & & & & & & & & & & 0.001 \\
\hline$n$ & & & & & & & & & & & \\
\hline$\%$ & & & & & & & & & & & \\
\hline Alcohol intake (units/week) & 14.4 & $12 \cdot 1$ & $7 \cdot 0$ & 7.9 & $5 \cdot 5$ & $6 \cdot 6$ & $3 \cdot 0$ & $4 \cdot 1$ & $2 \cdot 7$ & $5 \cdot 4$ & $<0.001$ \\
\hline Physical activity (active $v$. inactive) & & & & & & & & & & & 0.61 \\
\hline$n$ & & & & & & & & & & & \\
\hline$\%$ & & & & & & & & & & & \\
\hline Family history of diabetes & & & & & & & & & & & 0.42 \\
\hline$n$ & & & & & & & & & & & \\
\hline$\%$ & & & & & & & & & & & \\
\hline Total energy intake (kJ/d) & $8605 \cdot 8$ & $2272 \cdot 7$ & $8441 \cdot 1$ & $2261 \cdot 8$ & $8029 \cdot 8$ & $2265 \cdot 1$ & 7848.4 & $2072 \cdot 9$ & $7651 \cdot 1$ & $2399 \cdot 7$ & $<0.001$ \\
\hline Subcohort non-cases ( $n$ 3367) & & & & & & & & & & & \\
\hline$n$ & & & & & & & & & & & \\
\hline Age (years) & $57 \cdot 6$ & $9 \cdot 7$ & $58 \cdot 6$ & $9 \cdot 5$ & $58 \cdot 7$ & $9 \cdot 4$ & 59.5 & $9 \cdot 2$ & $59 \cdot 2$ & $9 \cdot 2$ & $<0.001$ \\
\hline Sex (men) & & & & & & & & & & & $<0.001$ \\
\hline$n$ & & & & & & & & & & & \\
\hline$\%$ & & & & & & & & & & & \\
\hline BMI $\left(\mathrm{kg} / \mathrm{m}^{2}\right)$ & $26 \cdot 7$ & 4.0 & $26 \cdot 3$ & $3 \cdot 7$ & $26 \cdot 2$ & 3.9 & $25 \cdot 9$ & $3 \cdot 7$ & $26 \cdot 0$ & $3 \cdot 8$ & $<0.001$ \\
\hline Height & $168 \cdot 2$ & $8 \cdot 7$ & $166 \cdot 9$ & $8 \cdot 8$ & $166 \cdot 3$ & $9 \cdot 1$ & $166 \cdot 0$ & $9 \cdot 0$ & $166 \cdot 2$ & 8.6 & $<0.001$ \\
\hline Weight & $75 \cdot 8$ & $14 \cdot 1$ & 73.5 & $12 \cdot 8$ & $72 \cdot 6$ & $12 \cdot 9$ & 71.6 & 11.9 & $72 \cdot 0$ & $12 \cdot 3$ & $<0.001$ \\
\hline Smoking status (current) & & & & & & & & & & & $<0.001$ \\
\hline$n$ & & & & & & & & & & & \\
\hline$\%$ & & & & & & & & & & & \\
\hline Alcohol intake (units/week) & $27 \cdot 9$ & 24.5 & $14 \cdot 1$ & $14 \cdot 0$ & $8 \cdot 0$ & $10 \cdot 5$ & $5 \cdot 6$ & $8 \cdot 6$ & $3 \cdot 1$ & 5.4 & $<0.001$ \\
\hline Physical activity (active $v$. inactive) & & & & & & & & & & & 0.4 \\
\hline$n$ & & & & & & & & & & & \\
\hline$\%$ & & & & & & & & & & & \\
\hline Family history of diabetes & & & & & & & & & & & 0.07 \\
\hline$n$ & & & & & & & & & & & \\
\hline$\%$ & & & & & & & & & & & \\
\hline Total energy intake (kJ/d) & $8451 \cdot 1$ & $2385 \cdot 1$ & $8325 \cdot 3$ & $2167 \cdot 7$ & $8226 \cdot 2$ & 2077.9 & $8022 \cdot 3$ & $1989 \cdot 3$ & $7698 \cdot 3$ & $2176 \cdot 9$ & $<0.001$ \\
\hline
\end{tabular}




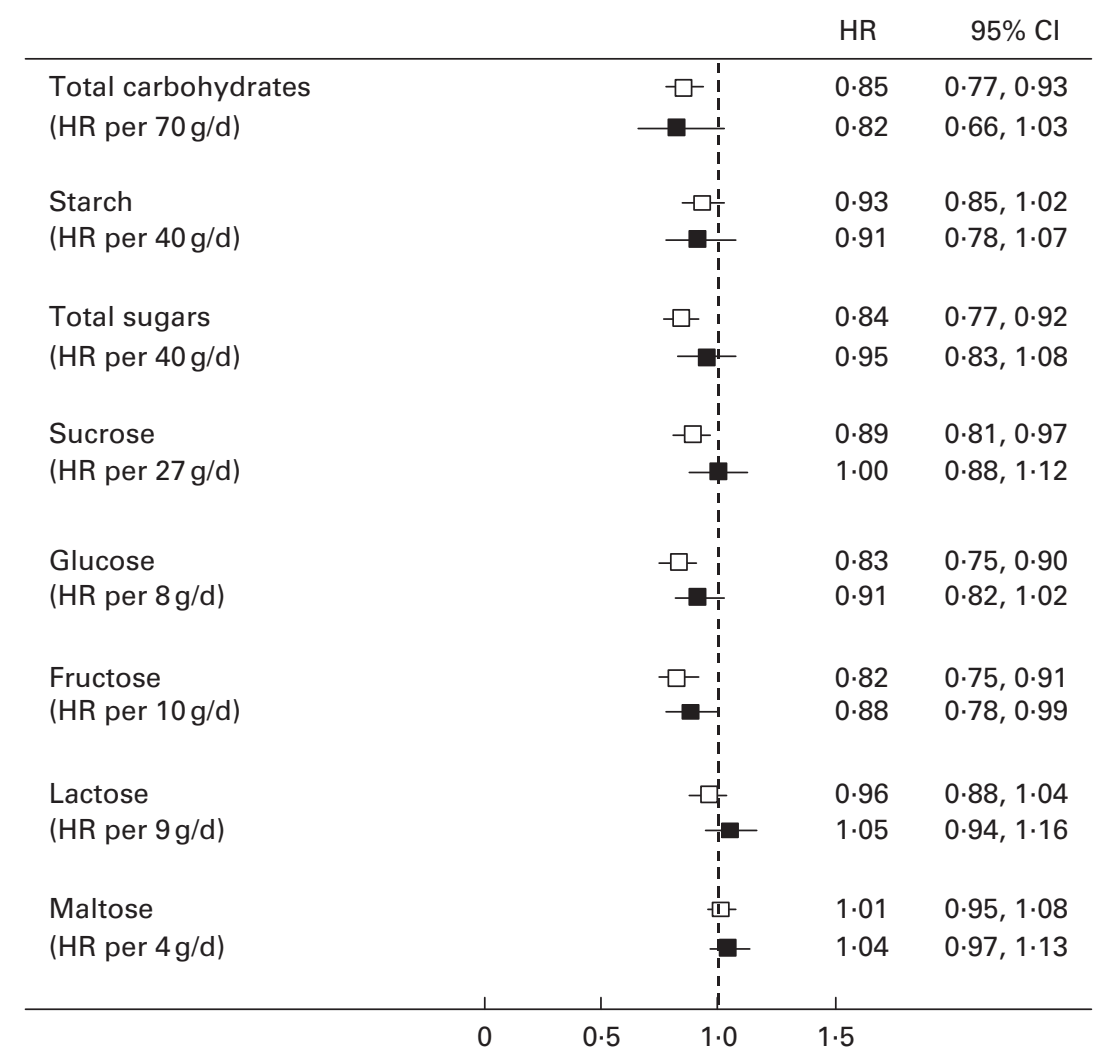

Fig. 1. Hazard ratios (HR) of type 2 diabetes per $1 \mathrm{SD}$ higher intake of each carbohydrate subtype with progressive adjustment for covariates using the residual method for energy adjustment. HR are presented for approximately 1 SD higher intake (g/d). Models (- $\square$ ) adjusted for age and sex. Models (- - ) additionally adjusted for total energy intake, BMI, family history of type 2 diabetes, cigarette smoking, alcohol intake, physical activity and level of education.

\section{Energy partition method for energy adjustment}

On applying the energy partition method, it was found that the net higher intakes of fructose and glucose were inversely related to diabetes risk. Multivariable HR of diabetes comparing the highest and lowest quintiles of intake were 0.72 (95\% CI $0.54,0.97 ; P$ for trend=0.01) for fructose and 0.68 (95\% CI 0.50, 0.93; $P$ for trend=0.01) for glucose. Sucrose, starch and other carbohydrate subtypes were not significantly related to diabetes risk.

\section{Sensitivity analyses}

When the analyses were repeated for cases diagnosed 5 years or more after recruitment or after excluding potential energy misreporters, there was no notable change in the findings (data not shown). In the analyses stratified by sex, the direction of the associations remained unchanged and was generally similar in men and women for all nutrients. Although there was no statistically significant effect modification by BMI (Wald test for interaction: $P=0 \cdot 19$ for glucose and $P=0.11$ for fructose), the inverse associations of fructose and glucose with diabetes risk were more pronounced in normal-weight (BMI $<25 \mathrm{~kg} / \mathrm{m}^{2}$ ) and overweight (BMI $25-29.9 \mathrm{~kg} / \mathrm{m}^{2}$ ) individuals, but subsided in obese individuals $\left(\mathrm{BMI}>30 \mathrm{~kg} / \mathrm{m}^{2}\right)$. There was no significant effect modification by family history of diabetes either (Wald test for interaction: $P=0.54$ for glucose and $P=0.51$ for fructose). However, the inverse associations were more pronounced in those without a family history of diabetes, but subsided in those with a family history of diabetes.

The main sources of fructose and glucose were fruits ( 41 and $29.5 \%$ ), drinks (16\% for both), sugar ( 9.5 and $15.5 \%$ ), cakes (9 and $10 \%)$ and vegetables (6 and 6.6\%). The association of fructose (or glucose) with diabetes risk did not vary by source of the nutrient (data not shown).

\section{Discussion}

In the present case-cohort study, we found that while holding total energy intake constant, higher intakes of sucrose and starch were not associated with the risk of diabetes, but an inverse association was observed between intakes of fructose and glucose and the risk of diabetes diagnosed, on average, 6 years later. While holding total energy intake constant, substitution of energy intake from fructose with other sources of energy, specifically SFA and protein, was inversely related to diabetes risk.

The results of previous prospective studies on the association between carbohydrate subtypes and the risk of diabetes are inconsistent. Different studies have reported decreased, increased or no association of diabetes risk with the same carbohydrate subtype ${ }^{(4,5,7-12)}$. Since the FFQ does not capture all sources of energy intake, the conflicting results might be explained by different FFQ administered in different studies capturing different sources of carbohydrate (e.g. extrinsic sugars $v$. intrinsic sugars) or capturing different diet- 


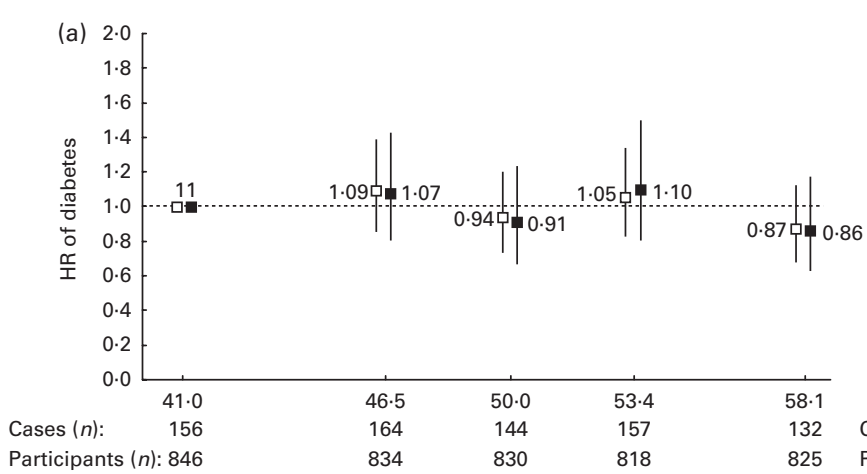

Percentage of total energy intake

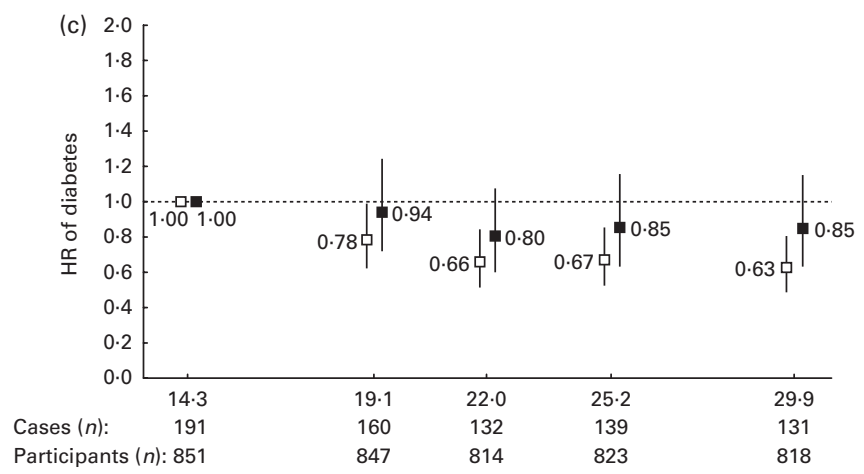

Percentage of total energy intake

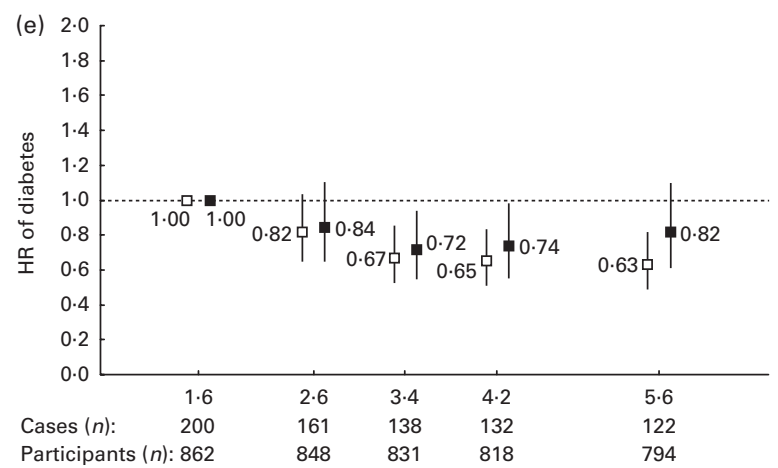

Percentage of total energy intake

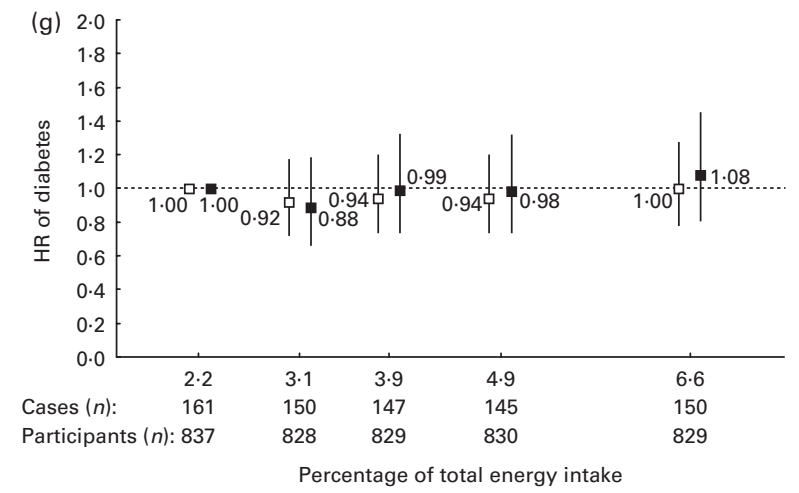

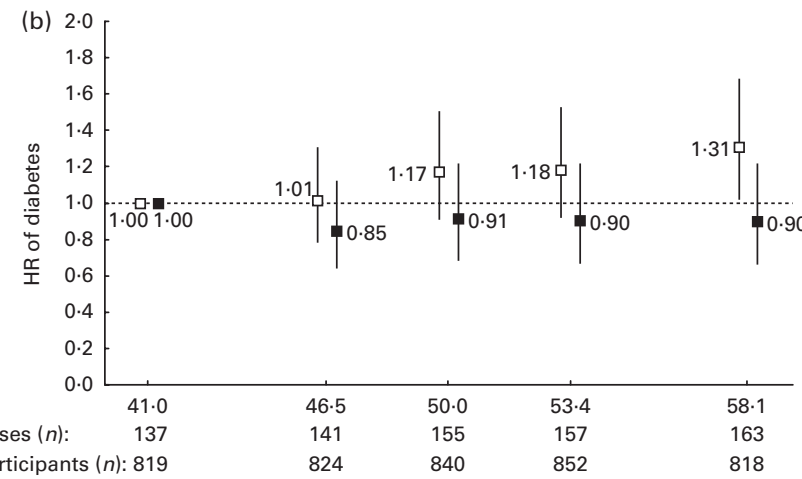

Percentage of total energy intake

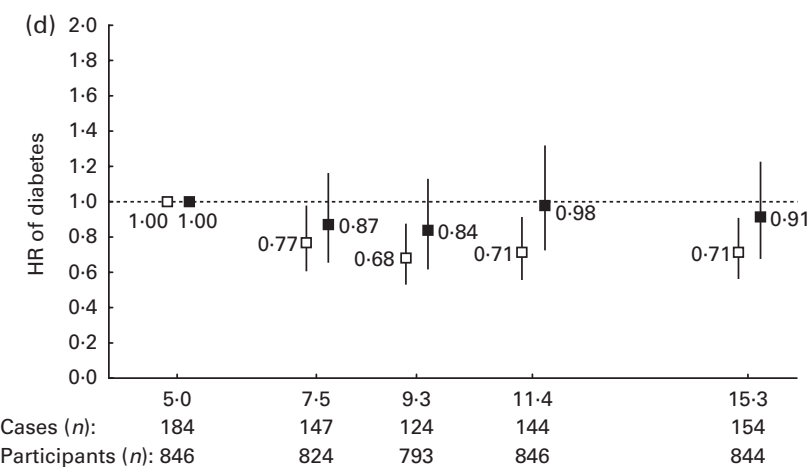

Percentage of total energy intake

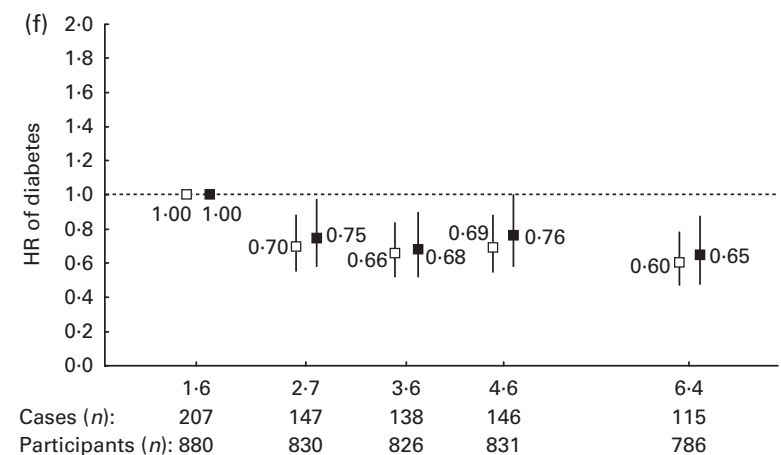

Percentage of total energy intake

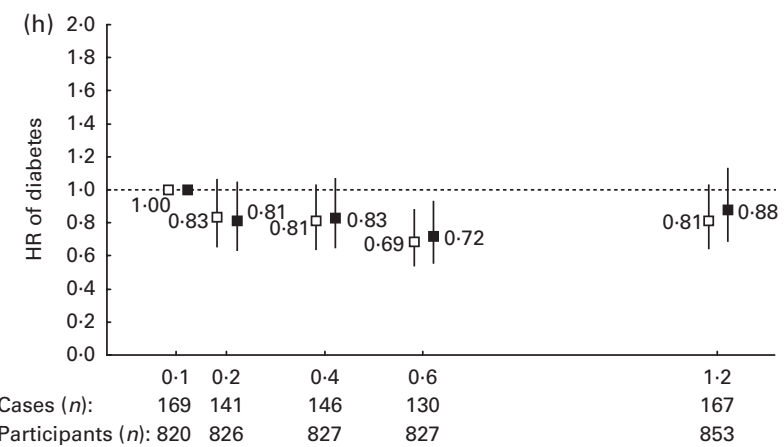

Percentage of total energy intake

Fig. 2. Hazard ratios (HR) of diabetes ((a) total carbohydrates, (b) starch, (c) total sugars, (d) sucrose, (e) glucose, (f) fructose, (g) lactose and (h) maltose) across the quintiles of percentage of energy intake from carbohydrate subtypes using the nutrient density method for energy adjustment. Models (占) adjusted for age and sex. Models ( $\$$ ) additionally adjusted for total energy intake, family history of type 2 diabetes, cigarette smoking, alcohol intake, physical activity, level of education and BMI. Numbers next to each box are the HR of diabetes. HR $(95 \% \mathrm{Cl})$ are plotted against median percentage of energy intake in the relevant quintile for each nutrient. 
Table 3. Hazard ratios (HR) of diabetes for substitution of $5 \%$ energy intake from total carbohydrates, fructose, glucose or sucrose with isoenergetic amounts of other energy-producing nutrients $\dagger$ (Hazard ratios and $95 \%$ confidence intervals)

\begin{tabular}{|c|c|c|c|c|c|c|c|c|}
\hline \multirow[b]{2}{*}{ Substitution with } & \multicolumn{2}{|c|}{$\begin{array}{c}\text { Total carbo- } \\
\text { hydrates }\end{array}$} & \multicolumn{2}{|c|}{ Fructose } & \multicolumn{2}{|c|}{ Glucoseł } & \multicolumn{2}{|c|}{ Sucrose } \\
\hline & $\mathrm{HR}$ & $95 \% \mathrm{Cl}$ & $\mathrm{HR}$ & $95 \% \mathrm{Cl}$ & $\mathrm{HR}$ & $95 \% \mathrm{Cl}$ & $\mathrm{HR}$ & $95 \% \mathrm{Cl}$ \\
\hline Other sugars§ & & - & 0.73 & $0.51,1.05$ & 0.76 & $0.49,1.18$ & 1.12 & $0.93,1.34$ \\
\hline Starch§ & & - & 0.74 & $0.54,1.03$ & 0.76 & $0.52,1 \cdot 12$ & 1.04 & $0.90,1.20$ \\
\hline Protein§ & 0.98 & $0.82,1.17$ & 0.72 & $0.51,1.03$ & 0.74 & $0.49,1.12$ & 0.99 & $0 \cdot 82,1 \cdot 19$ \\
\hline Total fat§ & 0.96 & $0.89,1.05$ & $0.72^{*}$ & $0.53,0.98$ & 0.73 & $0.50,1.07$ & 1.01 & $0.88,1.16$ \\
\hline SFA§ & 0.91 & $0.79,1.06$ & $0.69^{*}$ & $0.50,0.96$ & 0.70 & $0.47,1.04$ & 0.96 & $0.80,1 \cdot 15$ \\
\hline PUFA§ & $1 \cdot 16$ & $0.94,1.43$ & 0.85 & $0.59,1.22$ & 0.88 & $0.57,1.35$ & 1.17 & $0.94,1.45$ \\
\hline MUFA§ & $1 \cdot 13$ & $0.90,1.41$ & 0.82 & $0.57,1.19$ & 0.84 & $0.54,1.29$ & $1 \cdot 16$ & $0.90,1.48$ \\
\hline
\end{tabular}

${ }^{*} P<0.05$.

†The modelling strategy explained in footnote $\S$ (below) is used, with the exception of modelling substitution patterns with total carbohydrates, for which the term for energy intake from sugars or starch is not entered. Other nutrient terms are entered as described in footnote $\S$.

$\ddagger$ Energy intake from total carbohydrates (/fructose, glucose and sucrose) was entered into the model and adjusted for total energy intake, age, sex, BMI, smoking, alcohol intake, physical activity, family history of diabetes and level of education.

$\S$ In order to build substitution patterns, energy intakes from other nutrients were entered into the models as separate terms as follows: other sugars - separate terms for energy intake from starch, total fat and total protein; starch - energy intake from all sugars except fructose (/glucose or sucrose), total fat and total protein; protein - energy intake from all sugars except fructose (/glucose or sucrose), starch and total fat; total fat - energy intake from all sugars except fructose (/glucose or sucrose), starch and total protein; SFA - energy intake from all sugars except fructose (/glucose or sucrose), starch and total protein and energy intake from all fats except SFA; PUFA - energy intake from all sugars except fructose (/glucose or sucrose), starch and total protein and energy intake from all fats except PUFA; MUFA - energy intake from all sugars except fructose (/glucose or sucrose), starch and total protein and energy intake from all fats except MUFA.

ary patterns associated with the nutrients. Nevertheless, in a meta-analysis of ten published prospective studies including 11517 incident diabetes events, the highest to lowest quintiles of intake of sucrose were associated with a $15 \%$ lower risk of diabetes (relative risk 0.85, 95\% CI 0.75, 0.97) ${ }^{(22)}$.

The lack of association between the intake of total carbohydrates, starch or sucrose and the risk of diabetes is in line with the findings of clinical research in people with diabetes that total energy intake rather than type and source of dietary carbohydrates is important in glycaemic control ${ }^{(23,24)}$. On the other hand, clinical trials in people with diabetes have shown better glycaemic control with low-glycaemic index diets $^{(25)}$. Thus, starch or sugar as composite variables, independent of source, may not capture the aspect of carbohydrate quality that is relevant for glycaemic control.

The standard, residual and nutrient density methods for energy adjustment statistically model the effect of substitution of nutrients in an isoenergetic diet in which total energy intake is held constant ${ }^{(19,21)}$. Therefore, a lower risk of diabetes associated with fructose and glucose in the present study might reflect the effect of substitution of fructose (or glucose) with other sources of energy. In the EPIC-Norfolk study, we found that replacement of 5\% energy from SFA with an isoenergetic amount of fructose was associated with a $30 \%$ lower risk of diabetes and replacement of protein with fructose resulted in a non-significant $27 \%$ lower risk of diabetes. In the EPIC-Potsdam study ${ }^{(10)}$, replacement of 5\% energy intake from protein or PUFA with an isoenergetic amount of carbohydrates was associated with a $23 \%$ and a $17 \%$ lower risk of diabetes, respectively. Likewise, an increased intake of protein has been shown to be associated with an increased risk of diabetes ${ }^{(26)}$. However, replacement of SFA with total carbohydrates has not been shown to be significantly associated with a lower risk of $\operatorname{CVD}^{(27,28)}$, which highlights the importance of the 'type' of carbohydrate replacing SFA in developing health outcomes.

The models using the energy partition method for energy adjustment reflect the effect of net increase in energy intake from the nutrient without substitution with other nutrients. Yet again, we found an inverse association of the risk of diabetes with net higher fructose and glucose intakes. Therefore, the inverse association with diabetes risk might be related to higher intakes of fructose or glucose per se or might be related to factors specifically associated with their intake, such as particular micronutrients or particular dietary patterns that are related to a lower risk of diabetes. More specifically, fruits and vegetables are likely to account for the observed associations, as they are the main sources of fructose and glucose and have been shown to be inversely associated with the risk of incident diabetes ${ }^{(29)}$.

Our findings are in contrast with those of animal studies that have shown that feeding high-sucrose and high-fructose diets to rats induces diabetes and insulin resistance ${ }^{(30-32)}$. To the best of our knowledge, experimental evidence on humans is not conclusive ${ }^{(33)}$. Studies on normal and hyperinsulinaemic individuals have shown increased fasting glucose levels, insulin response and insulin resistance after consumption of diets with a higher percentage of energy intake from sucrose ${ }^{(34,35)}$ or fructose $\mathrm{e}^{(36)}$. On the other hand, in individuals with type 2 diabetes, high-fructose diets increase insulin sensitivity ${ }^{(37,38)}$. Moreover, in line with our findings, a systematic review and meta-analysis of clinical trials ( $n$ 18) has shown improved long-term glycaemic control with isoenergetic replacement of fructose with other carbohydrates in people with diabetes $^{(39)}$. Higher urinary excretion of fructose (a biomarker for fructose consumption) was associated with a lower risk of obesity in the EPIC-Norfolk cohort study ${ }^{(40)}$. The association of a lower risk of obesity with a higher intake of fructose is 
in line with and might provide some explanation for a similar association with diabetes. The contrast between our findings and what is expected from animal studies strengthens the idea that a lower risk of diabetes and obesity associated with fructose and glucose could be ascribed to particular dietary patterns (i.e. higher intake of fruits and vegetables) rather than to higher consumption of fructose itself.

The inverse association between carbohydrates and diabetes might be considered a consequence of the tendency of more obese people to under-report their carbohydrate intake. However, no association was observed with diabetes in obese individuals, while there was a significant inverse association of diabetes risk with fructose and glucose in lean participants. This suggests that potential under-reporting of carbohydrate intake in obese individuals is not likely to account for the observed associations. Moreover, excluding individuals with potential energy misreporting from the analyses did not change the results.

In the present study, we aimed to assess the effect of carbohydrate intake on the risk of diabetes independent of the effect of body size and total energy intake. We also adjusted for the potential confounding effect of physical activity and other known risk factors for diabetes. However, the effect of residual confounding by unmeasured or incomplete measured lifestyle factors is still possible.

To the best of our knowledge, this is the first study on the association between dietary intake of carbohydrate subtypes and the risk of diabetes using the food diary method for dietary assessment. Most epidemiological studies use FFQ as the method for dietary assessment due to its feasibility of administration and ability to capture long-term dietary habits. Completing food diaries is more costly, has greater participant burden and also captures the food intake over a short period of time. However, it has been suggested that food diaries may provide a more accurate measure of dietary intake than FFQ because they are prospectively recorded and are less subject to recall issues and individual tendencies to over-report or under-report specific items of the diet than are FFQ, are not limited to predefined items and can provide more detailed information on portion sizes ${ }^{(41,42)}$. The validity and reliability of $7 \mathrm{~d}$ food diaries have been assessed previously and compared with those of the FFQ and $24 \mathrm{~h}$ dietary recalls in the EPIC-Norfolk study ${ }^{(15)}$. In terms of day-to-day variation in diet and random measurement error, there were no significant differences in mean intake values obtained from the first (baseline) and second (administered at 18 months after recruitment) food diaries in the subset of participants with available data in the EPIC-Norfolk study ${ }^{(15)}$. In addition, when validated against serum vitamin $\mathrm{C}$ levels and $24 \mathrm{~h}$ urinary excretion of $\mathrm{N}, \mathrm{Na}$ and $\mathrm{K}$ as reference values, the $7 \mathrm{~d}$ food diary method was generally more repeatable and valid than the FFQ and $24 \mathrm{~h}$ recall methods ${ }^{(15)}$.

A limitation of the present study is that dietary data used for analyses were based on one measurement. Ignoring random day-to-day variation and changes in diet over time might have attenuated the observed association towards the null (regression dilution bias). Moreover, diet assessed once might not be representative of the usual diet. Not accounting for possible changes in confounding factors might result in residual confounding. However, we used the intake of nutrients in the present study, which is likely to be more stable across time than that of specific food groups.

In the EPIC-Norfolk study, the entire cohort was not biochemically screened for type 2 diabetes either at baseline or during follow-up. Therefore, there is the possibility for under-ascertainment of diabetes and therefore misclassification of the outcome, resulting in the attenuation of the observed association. However, rigorous case ascertainment methods were used to possibly capture all diagnosed cases of diabetes using multiple data sources and independently of active participation of study participants in follow-up visits or questionnaires. Moreover, there was a high specificity in ascertainment of diabetes, because confirmation of diagnosis of diabetes was either based on or verified by medical records. Hence, the misclassification of unidentified cases should not have biased the estimated risks. Another limitation is that a number of prospectively identified cases of diabetes might have had the condition undiagnosed at baseline. Repetition of the analysis on the subset of cases identified 5 years or more after recruitment to minimise the potential misclassification did not have a notable effect on the findings in terms of magnitude or statistical significance of the observed associations.

In summary, in the present study, we found that intakes of sucrose and starch were not related to the risk of diabetes and higher intakes of fructose and glucose were associated with a lower diabetes risk. This association might be due to higher intakes of glucose and fructose per se or might be related to other unmeasured nutrients associated with their intake or might reflect the true effect of the substitution of fructose with other nutrients, particularly SFA. The identification of dietary patterns associated with diabetes risk requires further investigation.

\section{Acknowledgements}

The authors thank all the study participants, general practitioners and the EPIC-Norfolk study team for their contribution. They also thank Ruth Keogh, MRC Biostatistics Unit, Cambridge, UK, and Stephen Sharp, MRC Epidemiology Unit, Cambridge, UK, for their help with statistical analyses. The EPIC-Norfolk study is supported by funding from the Medical Research Council and Cancer Research UK with additional support from the Stroke Association, British Heart Foundation, Research Into Ageing, and the Academy of Medical Science. S. A.-A. and R. C. are supported by the Gates Cambridge scholarship. Funding sources did not have a role in the design and conduct of the study, the collection, management, analysis and interpretation of the data, or the preparation, review and approval of or decision to submit the manuscript. S. A.-A. analysed the data and wrote the manuscript with the co-authors. R. N. L. and A. B. were responsible for data management and together with N. G. F. constructed the dataset for case-cohort analysis. N. P. analysed and coded the $7 \mathrm{~d}$ food diaries for derivation of the nutrients. K. T. K. and N. J. W. were principal investigators 
of the study and together with N. G. F. supervised this work. All authors provided helpful comments on the manuscript, reviewed and edited the manuscript and contributed to the discussion.

The authors declare that there is no conflict of interest associated with this manuscript.

\section{References}

1. Gillies CL, Abrams KR, Lambert PC, et al. (2007) Pharmacological and lifestyle interventions to prevent or delay type 2 diabetes in people with impaired glucose tolerance: systematic review and meta-analysis. BMJ 334, 299.

2. Brand-Miller JC (2004) Postprandial glycemia, glycemic index, and the prevention of type 2 diabetes. Am J Clin Nutr 80, 243-244.

3. Barclay AW, Petocz P, McMillan-Price J, et al. (2008) Glycemic index, glycemic load, and chronic disease risk a meta-analysis of observational studies. Am J Clin Nutr 87, 627-637.

4. Sluijs I, van der Schouw YT, van der AD, et al. (2010) Carbohydrate quantity and quality and risk of type 2 diabetes in the European Prospective Investigation into Cancer and Nutrition-Netherlands (EPIC-NL) study. Am J Clin Nutr 92, 905-911.

5. Villegas R, Liu S, Gao YT, et al. (2007) Prospective study of dietary carbohydrates, glycemic index, glycemic load, and incidence of type 2 diabetes mellitus in middle-aged Chinese women. Arch Intern Med 167, 2310-2316.

6. Schulze MB, Liu S, Rimm EB, et al. (2004) Glycemic index, glycemic load, and dietary fiber intake and incidence of type 2 diabetes in younger and middle-aged women. $A m \mathrm{~J}$ Clin Nutr 80, 348-356.

7. Hodge AM, English DR, O'Dea K, et al. (2004) Glycemic index and dietary fiber and the risk of type 2 diabetes. Diabetes Care 27, 2701-2706.

8. Meyer KA, Kushi LH, Jacobs DR, et al. (2000) Carbohydrates, dietary fiber, and incident type 2 diabetes in older women. Am J Clin Nutr 71, 921-930.

9. Janket SJ, Manson JE, Sesso H, et al. (2003) A prospective study of sugar intake and risk of type 2 diabetes in women. Diabetes Care 26, 1008-1015.

10. Schulze MB, Schulz M, Heidemann C, et al. (2008) Carbohydrate intake and incidence of type 2 diabetes in the European Prospective Investigation into Cancer and Nutrition (EPIC)-Potsdam Study. Br J Nutr 99, 1107-1116.

11. Montonen J, Jarvinen R, Knekt P, et al. (2007) Consumption of sweetened beverages and intakes of fructose and glucose predict type 2 diabetes occurrence. J Nutr 137, 1447-1454.

12. Barclay AW, Flood VM, Rochtchina E, et al. (2007) Glycemic index, dietary fiber, and risk of type 2 diabetes in a cohort of older Australians. Diabetes Care 30, 2811-2813.

13. Kim HS, Paik HY, Lee KU, et al. (1988) Effects of several simple sugars on serum glucose and serum fructose levels in normal and diabetic subjects. Diabetes Res Clin Pract 4, 281-287.

14. Day N, Oakes S, Luben R, et al. (1999) EPIC-Norfolk: study design and characteristics of the cohort. European Prospective Investigation of Cancer. $\mathrm{Br} J$ Cancer 80, Suppl. 1, 95-103

15. Bingham SA, Welch AA, McTaggart A, et al. (2001) Nutritional methods in the European Prospective Investigation of Cancer in Norfolk. Public Health Nutr 4, 847-858.

16. Welch AA, McTaggart A, Mulligan AA, et al. (2001) DINER (Data Into Nutrients for Epidemiological Research) - a new data-entry program for nutritional analysis in the EPIC-Norfolk cohort and the 7-day diary method. Public Health Nutr 4, 1253-1265.

17. Barlow WE, Ichikawa L, Rosner D, et al. (1999) Analysis of case-cohort designs. J Clin Epidemiol 52, 1165-1172.

18. Prentice RL (1986) A case-cohort design for epidemiologic cohort studies and disease prevention trials. Biometrika $\mathbf{7 3}$, $1-11$.

19. Kipnis V, Freedman LS, Brown CC, et al. (1993) Interpretation of energy adjustment models for nutritional epidemiology. Am J Epidemiol 137, 1376-1380.

20. Kipnis V, Freedman LS, Brown CC, et al. (1997) Effect of measurement error on energy-adjustment models in nutritional epidemiology. Am J Epidemiol 146, 842-855.

21. Willett WC, Howe GR \& Kushi LH (1997) Adjustment for total energy intake in epidemiologic studies. Am J Clin Nutr $\mathbf{6 5}$, 1220S-1228S.

22. Ahmadi-Abhari S \& Chowdhury R (2011) Dietary intake of carbohydrates and risk of type 2 diabetes: a systematic review and meta analysis. $J$ Epidemiol Community Health 65. A220-A221

23. Kelley DE (2003) Sugars and starch in the nutritional management of diabetes mellitus. Am J Clin Nutr 78, 858S-864S.

24. Franz MJ (2001) Carbohydrate and diabetes: is the source or the amount of more importance? Curr Diab Rep 1, 177-186.

25. Ajala O, English P \& Pinkney J (2013) Systematic review and meta-analysis of different dietary approaches to the management of type 2 diabetes. Am J Clin Nutr 97, 505-516.

26. Sluijs I, Beulens JW, van der AD, et al. (2010) Dietary intake of total, animal, and vegetable protein and risk of type 2 diabetes in the European Prospective Investigation into Cancer and Nutrition (EPIC)-NL study. Diabetes Care 33, 43-48.

27. Micha R \& Mozaffarian D (2010) Saturated fat and cardiometabolic risk factors, coronary heart disease, stroke, and diabetes: a fresh look at the evidence. Lipids 45, 893-905.

28. Siri-Tarino PW, Sun Q, Hu FB, et al. (2010) Saturated fat, carbohydrate, and cardiovascular disease. Am J Clin Nutr 91, 502-509.

29. Harding AH, Wareham NJ, Bingham SA, et al. (2008) Plasma vitamin $\mathrm{C}$ level, fruit and vegetable consumption, and the risk of new-onset type 2 diabetes mellitus: the European prospective investigation of cancer - Norfolk prospective study. Arch Intern Med 168, 1493-1499.

30. Daly M (2003) Sugars, insulin sensitivity, and the postprandial state. Am J Clin Nutr 78, 865S-872S.

31. Cohen AM, Teitelbaum A \& Rosenman E (1977) Diabetes induced by a high fructose diet. Metabolism 26, 17-24.

32. Goda T, Yamada K, Sugiyama M, et al. (1982) Effect of sucrose and Acarbose feeding on the development of streptozotocin-induced diabetes in the rat. J Nutr Sci Vitaminol (Tokyo) 28, 41-56.

33. Laville M \& Nazare JA (2009) Diabetes, insulin resistance and sugars. Obes Rev 10, Suppl. 1, 24-33.

34. Reiser S, Handler HB, Gardner LB, et al. (1979) Isocaloric exchange of dietary starch and sucrose in humans. II. Effect on fasting blood insulin, glucose, and glucagon and on insulin and glucose response to a sucrose load. $A m J$ Clin Nutr 32, 2206-2216.

35. Reiser S, Bohn E, Hallfrisch J, et al. (1981) Serum insulin and glucose in hyperinsulinemic subjects fed three different levels of sucrose. Am J Clin Nutr 34, 2348-2358.

36. Hallfrisch J, Ellwood KC, Michaelis OE, et al. (1983) Effects of dietary fructose on plasma glucose and hormone responses in normal and hyperinsulinemic men. $J$ Nutr 113, 1819-1826. 
37. Koivisto VA \& Yki-Jarvinen H (1993) Fructose and insulin sensitivity in patients with type 2 diabetes. J Intern Med 233, 145-153

38. Crapo PA, Kolterman OG \& Henry RR (1986) Metabolic consequence of two-week fructose feeding in diabetic subjects. Diabetes Care 9, 111-119.

39. Cozma AI, Sievenpiper JL, de Souza RJ, et al. (2012) Effect of fructose on glycemic control in diabetes: a systematic review and meta-analysis of controlled feeding trials. Diabetes Care 35, 1611-1620.
40. Bingham S, Luben R, Welch A, et al. (2007) Epidemiologic assessment of sugars consumption using biomarkers: comparisons of obese and nonobese individuals in the European prospective investigation of cancer Norfolk. Cancer Epidemiol Biomarkers Prev 16, 1651-1654.

41. Willett W (1998) Nutritional Epidemiology, 2nd ed. New York, NY: Oxford University Press.

42. Rothman K, Greenland S \& Timothy L (2008) Modern Epidemiology, 3rd ed. Philadelphia, PA: Wolters Kluwer Lippincott Williams and Wilkins. 